\title{
LAS DIATOMEAS DEL PLANCTON MARINO DE LAS COSTAS DEL BRASIL
}

\author{
(RECEBIDO $27 / V 11 / 55$ )
}

\section{F.C. Mïller-Melchers}

Poco se conoce del plancton de las costas del Este de Sud America. Las expediciones antarcticas bajaron casi todas por la costa africana y así se tienen varios trabajos sobre las diatomeas del plancton. El presente trabajo es resultado de la investigación de diez muestras de plancton recibidas del Instituto Oceanográfico de San Paulo. El estudio no pretende dar una reseña completa de todas las diatomeas de la costa brasilera. Para tal trabajo el material que estaba a mi disposición no era bastante diversificado y tanpoco abarcaba las costas del Norte y Sur del Brasil.

Las muestras que se investigaron se componian de tres colecciones. Las primeras muestras, 1 al 10 me fueron facilitadas por intermedio del Sr. J. de Paiva Carvalho del Instituto Oceanográfico de San Paulo a quien le doy mi muy sentido agradecimiento. El material se compone de muestras del Canal de Santos y el resto de muestras costeras y lagunares de la costa de Cananéia. Estas muestras han demostrado un interesante conjunto, especialmente entre las especies de los Coscinodiscus y Chaetoceros. Entre los últimos está el Chaetoceros furcellatus que se conoce de aguas mas bien frias, como también una forma que tiene parecido al Ch.karianus (dudoso?) que también proviene de agua fria. En estas muestras se encontraron algunas especies poco conocidas y nuevas. 
La segunda serie tengo que agradecer al Sr. J.Soriano, es de la barra de Rio Grande do Sul. Esta muestra es sumamente interesante como demuestra todavia cierta influencia del plancton uruguayo, seguramente arrastrado a las costas brasileras por la corriente fria de Las Malvinas (Falkland Isl. Current). Hay buent cantidad de diatomeas planctonicas uruguayas y de otra parte muchas brasileras que no estan conocidas en las costas uruguayas, como por ejemplo Campy lodiscus Daemelianus y Actinoptychus splendens. Este último aparece recién en las costas atlanticas de la Prov. de Ruenos Aires. Las grandes Biddulphias laevis abundan, también desconocidas en aguas uruguayas. Ciertas formas como Actinocyclus platensis, Coscinodiscus punctiger y Cosc. Rothii formas típicas uruguayas siguen ahora casi por toda la costa hacia el Norte.

La tercera serie procede de pescas tomadas por el Ingeniero J. Weiher a bordo de la motonave "Sta.Catarina". Se trata de muestras tomadas por la bomba de agua de mar durante el viaje para Europa. Estas muestras fué un experimento que por gentileza del Ing. Weiher se pudo realizar. La red planctonica fué colocada en el hidrante de la bomba y se filtraba el agua durante unas tres a cuatro horas. Así se ha conseguido interesantes muestras que han suplementado las muestras de Santos y Cananéia.

Las diversas muestras de plancton demuestran regiones bastante bien definidas con tipos de diatomeas guia. Al Sur - Rio Grande do Sul - la provincia de agua fria a fria templada. De aquí hasta $20^{\circ} \mathrm{S}$ - Rio de Janeiro - la región subtropical a tropical y la tercera región tropical y equatorial oceanica con tipos como As terolampra marylandica etc. Como dicho al principio este estudio es un tanteo, basado sobre pocas muestras pero ya se da una idea de lo que podrá esperarse de investigaciones más intensas con muestras más grandes y en diversas estaciones del año. Siguiendo más al Norte y pasando el ecuador aumentan los dinoflagelados y especialmente los radiolarios para casi tener la predominancia en el oceeno abierto, como me han demostrado las muestras proveriendo desde las Rocas San Pablo hasta las islas de Cabo Verde.

El modo de investigación fué de observar primero las muestras de plancton en estado natural, sin colorear ó oxidar. Esto era necesario para determinar los generos de Chaetoceros y de los demás tipos de silificación escasa. Para las diatomeas discoides de los generos Coscinodiscus, Actinoptychus, Actinocyclus, etc. y los demás individuos de sílice más resistentes fueron hechas preparaciones del material oxidado con permanganato de potasa y peróxido 
de hidrógeno. En algunos casos de plancton muy transparente he usado el violeta de metilo para colorear, para no perder tipos hialinos. Las preparaciones que fueron hechas de todas las pescas fueron montadas en Sirax y Alkarina. Para ciertos casos se usó Estoraque y Hyrax. Una serie de preparaciones de las 10 muestras de Santos y Cananéia fueron mandadas al Sr. J. de Paiva Carvalho.

Guando habia terminado la investigación de las diez muestras me parecia que seria interesante de comparar al mismo tiempo el conjunto de diatomeas brasileras con los del plancton uruguayo y argentino. Los interesantes trabajos del Dr. J.Frenguelli dan una buena reseña de la costa argentina. Además el Prof. E. Balech me ha facilitado material de aguas argentinas. A esto agregué algunas pescas tomadas por mí en Necochea, Prov. B.A., Argentina.

Sobre la costa uruguaya he coleccionado plancton durante los últimos diez años y durante todos los meses del año. Se juntó un extenso material. La investigación sigue. Los datos de esta investigación ya están prontos para publicar. Basandomesobre el material brasilero he anotado los datos del Uruguay y de la Argentina al lado de cada especie brasilera. No han sido tomado en cuenta otras especies uruguayas ó argentinas, esto quedará para un trabajo futuro. En algunos casos van datos sobre la frecuencia de la especie ó datos sobre alguna particularidad. No se pudo ampliar demasiado para evitar un trabajo sumamente voluminoso. Siento no haber podido conseguir muestras de plancton de la parte norte de la costa brasilera - mis muestras terminan mas ó menos a la altura de las Rocas de San Pablo.

Muestras del Instituto Oceanográfico de San Paulo

$$
\begin{aligned}
& \text { No } 1 \text { - 4/ III/1950 - Canal de Santos. Superficie. } \\
& 2 \text { - 22/ III/1950 - " " " } \\
& 3-27 / \text { III/1950 - " " n }
\end{aligned}
$$

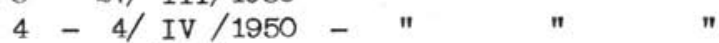

$$
\begin{aligned}
& 5 \text { - 22/ II/1949 - Mar de Trapandé. Litoral sul do Estado. } \\
& 6 \text { - 3/VIII/1949 - Rio Baguaçú. Litoral sul. } \\
& 7 \text { - 1/ IX/1949 - Rio Batatal. " " } \\
& 3 \text { - 26/X / } / 1949 \text { - Mar de Cubatão. } \\
& 9 \text { - 17/ I /1949 - Canal de S.Sebastiâo. Litoral norte, Praia } \\
& \text { da Fazenda. } \\
& 10 \text { - 28/ III/1940 - Baía de Santos. Ilha das Palmas. } \\
& \text { № } 329 \text { - Rio Grande do Sul. Muestra de fondo sobre la barra. } \\
& \text { J.Soriano leg. Octubre } 1952 .
\end{aligned}
$$



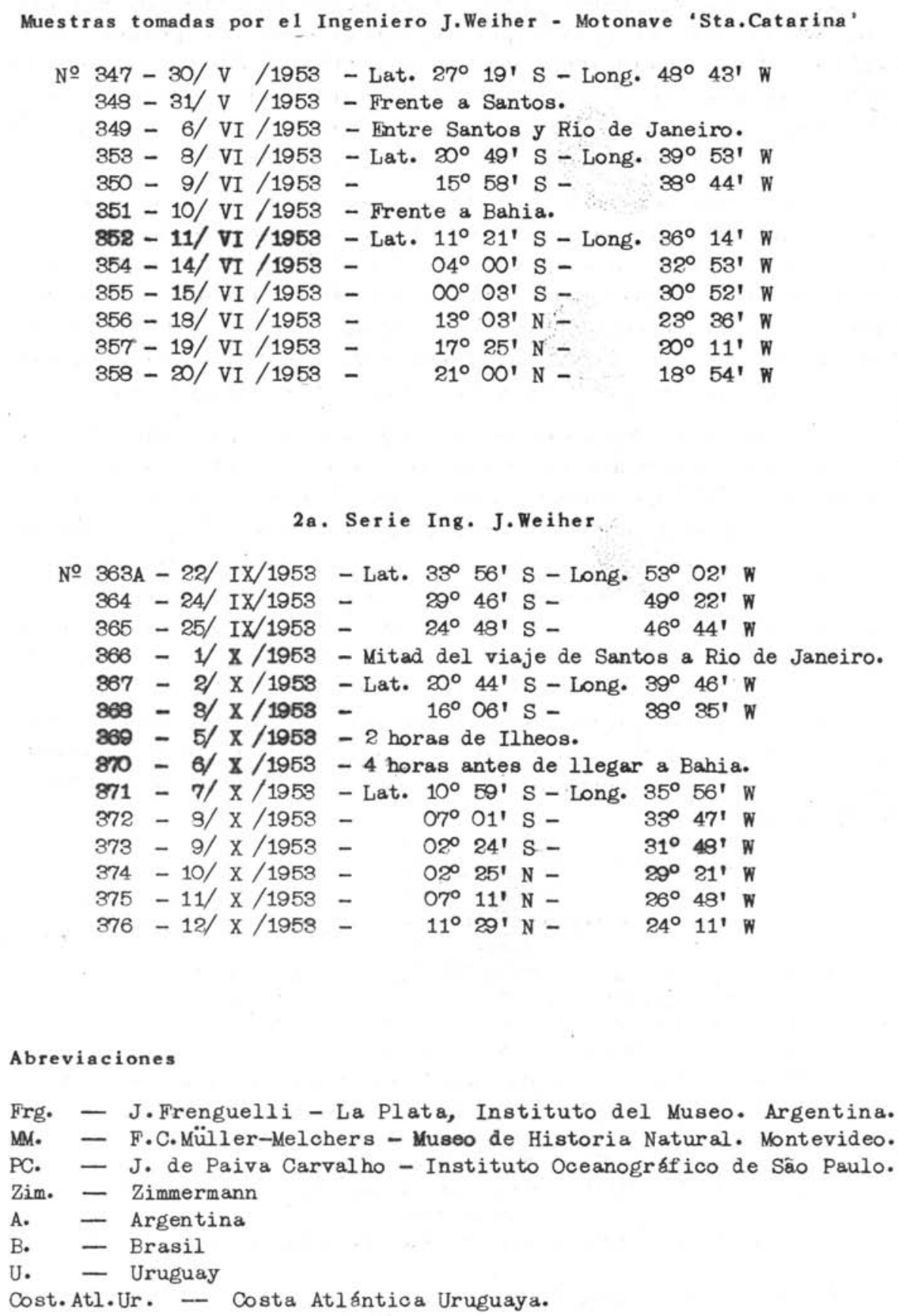


\section{ESPECIES INVESTIGADAS}

Achnanthes brevipes Agardh

syst.Alg., p. 1 (1824)

ZIMMERMANN Ach.subsessilis, p. 133(1913)
A. Frg. p. $263(1930)$
B. MM. 9.370 .
U. MM. Atlántida, Piriapolis, Punta del Este.

Achnan thes longipes Agardh

consp.critic., p. $58(1832)$
A. Frg. p. $233(1944)$
B. MM. 9.370 .
U. MM. La Paloma.

Actinocyclus crassus van Heurck

syn.Diat.Belg., p. $215(1880-85)$

ZI MUMERMANN , p. 66 (1913)

A. Frg. p. 327 (1938)

B. MM. 1. 334. 366. 368. 369.

U. MM. Atlántida.

Actinocyclus Ehrenbergi Ralfs

Pritchard Infus., p. 834 (1861)

ZI IMERMANN, p. 6 (1913)
A. Frg. p. 32 (1931)
B. MM. 7. 8. 334. 366. 370, 371.
U. MM. Costa Atlántica Uruguaya.

Actinocyclus Ehrenbergi var. moniliformis Ralfs Pritchard Infus., p. 834 (1861)

ZIMUERMANN, p. 8 (1913)
A. Frg. p. 298 (1930)
B. MM. 5. 334. 365.
U. MM. Cost.Atl.Ur. 
Actinocyclus platensis Müller-Melchers New and little known Diatoms (1954), p. 4, lám.111, fig. 1,2,3.
A. Frg. p. 523 (1928) bajo Acyclus Barkleyi
MM. Atlóntico Sur Argentino, 389.
B. MM. 1. 9. 334. 365 .
MM. Rio Grande do Sul - barra.
U. MM. p. 4 (1954)

Actinoptychus campanulifer A.Schmidt (1875)

A. Schmidt Atlas, $X X \mid x$, fig. 13, 14, 15.

ZIMUERMANN, p. 11 (1913)

LонMAN. К. E., 1941, p. 79 , plate 16, fig. 8

müller-melchers, Comun. 1954, p. 3
A. -
B. MM. 1. 4. 5. 9. 334. 366.
U. --

Actinoptychus senarius (Ehrenberg) Ehrenberg 1843

Berl.K.Akad.Wiss.Phys.Abh., p. 400, pl. 1, fig. 27 (1841)

Actinocyclus undulatus Kützing. Die kies.Bacill. oder Diat., p. 132, pl. 1, f. 44 (1844).

Actinoptychus undulatus (Kützing) Ralfs en Pritchard.

Actinoptychus undulatus (Bailey) Hustedt Die Kieselalgen, Teil I, p. 475, f. 264 (1920).

Act inoptychus senarius (Ehrenberg) Ehrenberg - Hendey, N.I. Southern Seas Discovery Rep., p. 271 (1937).

Actinoptychus senarius (Ehrenberg) Ehrenberg - Lohman, K. E. Geol. and Biol. North Atlant. Deep Sea Cores, p. 80 (1941). Actinoptychus senarius (Ehrenberg) Ehrenberg - Hustedt, Fr. Marine Littoral Diatoms of Beaufort N.C. (1955).

ZIMMERMANN, p. 6 (1913) debajo Actinoptychus undulatus

A. Frg. p. 156 (1924)

в. MM. 7. 8. 334. 366. 370. 371.

U. MM. Cost.Atl.Ur.

Actinoptychus splendens (Shadbolt) Ralfs Ralfs en Pritchard Infus., p. 840 (1861) 
ZIMUERMANM, p. 70 (1913)
A. Frg. p. 302 (1930)
B. MM. 1. 4. 5. 9. 334. 351. 352. 356.
U. MM. f6sil

Actinoptychus vulgaris Schuhmann

$$
\text { Preuss.Diat., p. } 64 \text { (1867) }
$$

f. 'polymera

f. octanario
A. Frg. p. 521 (1928)
B. MM. 4. 351. f. octanaria
U. Ma. Atlántida

Amphiprora pulchra Bailey

Microscop.obs., p. 38 (1860)
A. Frg. p. 291 (1941)
B. MM. 1. 5. 8 .
Zim. Itaparica
U. -

Asterionella japonica Cleve

Cleve \& Möller Diat., № 307 (1878)
A. Frg. p. 510 (1928)
B. MM. 5 .
PC. p. 36 (1950)
U. MM. Cost.Atl.Ur.

As terolampra marylandica Ehrenberg Ber.Berl.Akad. (1844), p. 76 (1845)
A. -

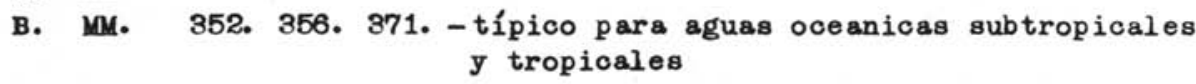
U. -

As teromphalus heptactis (Brébisson) Ralfs Brébisson Bull.Soc.Lin., Vol. 2, Spatangidium heptactis (1857) Ralfs Pritchard Infus., p. 838 (1861) 

A. -
B. MM.
4. 367 .
U. -

As teromphalus Hookerii Ehrenberg (Lám. I, fig. 1) Mon.Ber.Ak., p. 200 (1844)
A. -
B. MM. 5. 9. 334. - oceanico, raro
U. MM. plancton Cost.Atl.Ur., raro

\section{Aulacodiscus Kittoni Arnott \\ Pritchard Infus., p. 844 (1861)}
A. -
B. M. 354. - a la altura de Fernando Noronha
U. -

Bacteriastrum delicatulum Cleve

Fifteenth An.Rep.Fish. Board of Scotland, p. 298 (1897)

FARia cunha, p. 88 (1917), B. furcatum

Cunna Fonseca, B. furcatum, p. 102 (1918)
A. -
B. MM. 1. 5. 7. 8. 367.
U. MM. Atlántida

Bacterias trum hyal inum Lauder

Trans.Micr.Soc.N.S., Vol. 12, P. 6 (1864)
A. Frg. p. 543 (19æ8), Bact.varians
MM. Atlántico Sur Argentino
B. M. 5. 7. 8 .
PC. p. 34 (1951)
U. MM. Cost.Atl.Ur.

Bacteriastrum varians Lauder New Diatoms, p. 8 (1864)
A. -
B. MM. 1. 370.371 .
U. - 
Biddulphia an tedi luviana var. excava ta Frenguelli (Lám. I, fig. 7) Cuma \&onseca, p. 102 (1918), B.vesiculosa
A. Frg. p. 212, XV, fig. 2/3 (1945)
B. MM. 9 .
U. MM. La Paloma, Punta del Este

Biddulphia chinensis Gréville Descr. New and Rare Diat. (1866)

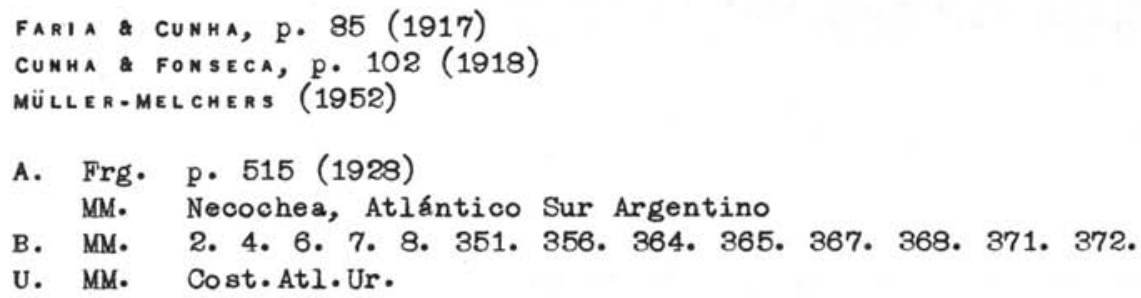
A. Frg. p. 514 (1928)
B. MM. 2. 4. 5. 6. 7. 8. 351. 364. 365.
PC. p. 34 (1951)
U. MM. Cost.Atl.Ur. 
Biddulphia pulchella Gray Nat.Arr.Brit.Plants, Vol. 1, p. 294 (1821)

Z I MMERMAN N P. 56 (1913)

FAria Cuma, B.biddulphiana, p. 84 (1917)
A. -
B. MM. 4. - Rio de Janeiro
U. MM. La Paloma

Biddulphia regia (Schultze) Ostenfeld Meddel.Komm. Havunders., Bd. 1 (1908)
A. -
B. MM. 7. 8. 372. 373.
U. -

Biddulphia rhombus (Ehbg.) Wm.Smith syn.Brit.Diat., Vol. II, p. 49 (1856)

Z I MUE RMANN, p. 65 (1943)

FARIA CUNHA, P. 85 (1917)

cunha fonseca, p. 102 (1918)
A. Frg. p. 513 (19æ8)
B. MM. 2. 4. 5. 7. 344 .
U. MM. Atlántida

Biddulphia roperiana Gréville Calif.Guano, p. 163 (1859)
A. Frg. p. 306 (1930)
B. MM. 5. 9.344 .
U. MM. Cost.Atl.Ur.

Biddulphia tuomeyi (Bailey) Roper Trans.Micr.Soc., Vol. 7, p. 8 (1859)

ZI MUE EMANN, P. 56 (1913)

FARIA CUMHA, B.tridens, p. 85 (1917)
A. -
B. MM. 1. 2. 4. 5. 334. 376.
U. MM. muy raro 
Campy lodiscus Daemelianus Grunow (Lám. I, fig. 2 y 3 ) Schmidt Atlas, pl. 17, fig. 11.
A. -
в. MM. 1. 2. 4. 352.
v. -

Campylodiscus Hodgsoni $i$ Wm. Smith Brit.Diat., pl. 6, fig. 53.

A.s. Atlas, pl.53, fig. 5 .
A. -
B. MM. 356 .
U. -
Cerataulus Smithii Ralfs
Pritchard Infus. (1861)
FArIA * CUNha, p. 84 (1917)
A. -
B. MM. 5 .
U. --

Chae toceros affinis Lauder (Lám. I, fig. 4)

Trans.Micr.Soc.N.S., Vol. XII, p. 68 (1864)

FARIA \& CUNAA, p. 83 (1917), Ch.Schüttii

Cunha a fonseca, p. 102 (1918), Ch.Schüttii

karstem atl.ocean como Ch.Ralfsii, p. 168, XXXIJ], fig. 17/18
A. Frg. p. $223(1939)$
B. MM. 7. 8. 368. con esporos 370. 372. 373. - las setas termi- nales son muy abiertas, parecido a los de Karsten. $05^{\circ} 06^{\prime} 02^{\prime \prime} \mathrm{S}-09^{\circ} 58^{\prime} 06^{\prime \prime} \mathrm{W}$, Victoria (Costa Africana) descrita como Ch. Ra1fsii
U. MM. p. $162(1949)$

Chae toceros apendiculatus Müller-Melchers New and little known Diatoms (1954), p. 6, pl. V, fig. 1, 2, 6 .
A. -
B. MM. 5. 7. 9.
U. MM. (1954) 
Chae toceros brevis Schütt

Ber.Deut.Bot.Ges., Vol. 13, p. 38 (1895)
A. -
B. MM. 7. 365 .
U. MM. p. 162 (1949)

Chae toceros coarctatus Lauder Trans.Micr.Soc.N.S., Vol. XII, p. 79 (1864)

FARIA CUNHA, p. 83 (1917)
A. -
B. MM. 352. 370. 387 .
U. MM. p. 164 (1949)

Chae toceros compressus Lauder Trans.Micr.Soc.N.S., Vol. XII, p. 78 (1864)

FARIA CUNHA, P. 83 (1917)
A. Frg. p. 551 (1928)
B. MM. 7. 8. 364. 365. 366, 367, 368, 370.
v. MM. p. 162 (1949)

Chae toceros confertus Müller-Melchers New and little known Diatoms, p. 7, VIl, fig. 1 (1954)
A. -
B. MiM. 5. - solamente una valva terminal
U. MM. (1954)

Chae toceros constrictus Gran Norske Nordhav Exp., p. 17 (1897)
A. -
B. MM. 368. 370. 372 .
U. MM. Cost.Atl.Ur.

Chae toceros curvisetus $\mathrm{Cleve}$

vidensk.udb. Kanonbaad.Haucks.Tog., p. 55 (1889)

FARIA \&UNHA, p. 84 (1917) 

A. Frg. p. 546 (1928)
M. Atlántico Sur Argentino
B. MM. 6. 7. 8. 10. 365. 368. 372.
PC. p. 35 (1951)
U. MM. p. $162(1949)$

Chae toceros debilis Cleve

Bih.Kongl.Sv.Vet.Ak.Hand1., Vol. XX, p. 13 (1894)

A. Frg. lám. X, fig. 5 (19æ8) bajo Ch.curvisetus

MM. Atlántico Sur Argentino

B. MM. 2. 8 .

U. MM. p. $162(1949)$

Chae toceros decipiens Cleve

Bih. Kongl.Sv.Vet.Ak. Handl., Vol. 1, p. 11 (1873)
A. Frg. p. $553(1928)$
MM. Atlántico Sur Argentino
B. MM. 365 .
U. MM. p. 162 (1949)

Chae toceros densus Cleve

seas.Distr.At 1. Plankton organ., p. 299 (1901)
A. -
B. MM. 365. - raro
U. MM. Oceano Atlántico Uruguayo, 22 millas al sur de la Paloma, Op. 66, Mayo 51, pescado por Dr. F. de Buen.

Chae toceros didymus Ehrenberg

Ber.Berl.Akad., p. 75 (1846)

FARIA \&UNHA, p. 83 (1917) v. ang1icum
A. Frg. p. 547 (1928)
B. MM. 2. 4. 7. 8. 365. var. anglica, 368. 370 .
U. MM. p. 162 (1949)

Chae toceros diversus Cleve

Bih. Kongl.Sv.Vet.Ak.Hand1., Vol. 1, p. 9 (1873)
A. -
B. MM.
4. 364. 367. - tropical ?
U. - 
Chae toceros lorenzianus Grunow

Verh.Zool.Bot.Ges.Wien, Vol.13, p. 157 (1863)

FARIA \& CUNHA, P. 83 (1917)
A. Frg. p. 222 (1939)
MM. Oceano Atléntico Argentino
B. MM. 5. 7. 8. 364 .
U. MM. p. $162(1949)$

Chae toceros furcellatum Bailey

Amer.Jour.Sc., Ser. 2, Vol. 22 (1856)

Cleve arunow, Arkt.Diat., p. 120, fig. 136 (1880)
A. -
B. MM. 4. 6. 7. 8 .
U. -

Conocido del Atlántico Norte y Arctico, no conocido en la Antarctica. Según Gran, Ciudad del Cabo.

Chae toceros ? Kariana ? Grunow

Cleve \& Grunow, Arkt.Diat., p. 120, fig. 135 (1880)

Espeoie parecida a Kariana, junto con Ch.curvisetus podría ser también una forma de esta última?
A. --
B. MM. 7. - frecuente
v. -

Chae toceros pseudocurvisetus Mangin

Bull.Soc.Bot. de France, Vol. 57, p. 350 (1910)
A. -
B. MM. 6. 8. 368. 372 .
v. MM. Atlóntida

Chae toceros peruvianus Brightwell Quart.Jour.Micr.Soc., Vol. IV, p. 107 (1856)

FARIA \&UNA, p. 83 (1917)
A. -
B. MM. 1. 4. 5. 7.
U. MM. p. 168 (1949) 
Chae toceros rostratus Lauder

Trans.Micr.Soc., N.S., Vol. XII, p. 79 (1864)
A. Frg. p. 554 (1928)
B. MM. 365. 368. 372 .
U. MM. p. 166 (1949)

Chae toceros seiracanthus Gran

Norske Nordhav.Exped., p. 21 (1897)

A. Frg. lám. X, 6. 7. (1928)
B. MM.
U. MM. p. $163(1949)$

Climacosphenia moniligera Ehrenberg Abh.Berl.Akad., p. 411 (1841)

ZI MUE RMAKN, C1.elongata, p. 137 (1913)
A. -
B. MM.
4. 8. 9.
U. --

Cocconeis scutellum Ehrenberf

Infus., p. 194 (1838)

ZI MUERMANN, P. 132 (1913)
A. Frg. p. $267(1930)$
B. MM. 9 .
U. MM. Atlántida

Core thron criophilum Castracane

Challenger, p. 85, xxI, fig. 14 (1886)
A. Frg. p. 332, lám. VI, fig. 3 (1938a)
B. MM. 7 .
PC. bajo hystrix Cleve
U. MM. Atlántida, raro en invierno. Es un tipo diferente comple- tamente a las formas antarcticas. Parecido a la figura en Gran, Nord. Plancton fig. 70, p. 57. Las sedas no tienen ganchos. Lo más parecido es la figura de Frenguelli, aquí algunas sedas tienen ganchos. Parece que es una especie de aguas frías hasta templadas.


Coscinodiscus apiculatus var. ambigua Grunow

Denkschr.Akad.Wiss.Wien, vol. 48, p. 75 (1884)
A. MM. Atlántico Sur Argentino
B. MM. 5. 352. 363A. 367. 370. 372.
U. MM. en el Coscinodiscus plancton del verano

Coscinodiscus asteromphalus Ehrenberg

Ber.Berl.Akad., p. 77 (1844)

ZIMUERMANM, P. 67 (1913)
A. Frg. 534. (1928)
MM. 397.
B. MM. 1. 2. 352. 363A. 364. 365. 366. 367. 368. 369. 370. 372. 374. 375. 376. 377.
U. MM. Atlántida

Coscinodiscus brasiliensis n.sp. (Lám. I, fig. 5)
A. -
B. MM.
5. 9 .
U. -

Valva discoide, bajando ligeramente en el borde. Areolación de perlas finas radiales. Los radios no salen del centro mismo, sino del borde de una área central. Esta área, apartada por una linea hialina de 108 radios contiene unas perlas irregularmente diseminadas. Los radios están en partes bifurcadas, el nuevo radio sale de adentro de la bifurcación. Especie no rara.
Díámetro de la valva
Perlas $14 / 15$ a $16 / 17$ en
$0,074 \mathrm{~mm}$
Radios en el borde 15 en
$0,01 \mathrm{~mm}$
Apiculi 2 en el borde
$0,01 \mathrm{~mm}$
$0,01 \mathrm{~mm}$

Coscinodiscus commutatus Grunow

Fr. Josef Land, p. 79 (1884)
A. Frg. p. $323(1941)$
B. MM. 363A. 365. 368. 372. 374. 375. 356.
U. MM. Atlántida

Coscinodiscus concinnus W. Smith

Syn. Brit. Diatoms, Vol. II, p. 85 (1856) 
ZIMUERMAN N, P. 10 (1913)
A. Frg. p. 537 (1928)
MM. Cabo Ines, Tierra de Fuego
в. MM. 7. 10. 351. 356. 364. 365. 367. 368. 369. 370. 373.
U. MM. En plancton del verano - raro en invierno. Cost.Atl.Ur.

Coscinodiscus curvatulus Grunow en A.Schmict Atlas, pl. 57, fig. 33 (1878)
A. Frg. p. $527(1928)$
B. MM. 9. 356. 364. 365. 368. 369. 370. 375.
U. MM. Costa Atlántica

Coscinodiscus cycloteres Castracane 1886, lám. XXII, fig. 8, p. 161
A. -
B. MM. 370. 374. - Especie muy parecida en areolación y tamaño a la especie de Castracane quien la describe como del

U. MM. Se han encontrado valvas muy parecidas en raras veces.

Coscinodiscus decrescens Grunow

$$
\text { A.S. At las, lám. 61, fig. 7-10 (1878) }
$$
A. -
B. MM. 5. 351. - raro
U. MM. raro

Coscinodiscus devius Schmidt

A.Schmidt At las, pl. LX, fig. 1-4
A. MM. $38^{\circ} 23^{\prime} \mathrm{S}-57^{\circ} 08^{\prime} \mathrm{W}$
B. MM. 1. 351. 366. 368. 370. 372.
บ. -

Coscinodiscus divisus Grunow (Lám. I, fig. 8)

Schneider Natur.Beitr.z. Kent.d. Kaukasusländer, p. 125 (1878)
A. Frg. p. $526(1928)$
B. MM. 4. 5. 365. 367. 359 .
U. MM. Atlántida 
Coscinodiscus excentricus Ehrenberg Abh.Berl.Akad., p. 146 (1839)

ZI IMAERMANN, p. 8 (1914)

FARIA \& CUNHA, P. 80 (1917)

Cunha \&onseca, p. 102 (1918)

paiva carvalho, p. 31 (1950)
A. Frg. p. 528 (1928)
MM. p. 322 (1951)
в. MM. 334. 351. 356. 364. 365. 366. 368. 376.
PC. p. 31 (1951)
u. MM. Cost.Atl.Ur.

Coscinodiscus excentricus var. micropora Grunow

Cleve \& Möller, no 114
A. Frg. p. 520 (1928)
B. MM. 5 .
u. -

Coscinodiscus gigas Ehrenberg Abh.Berl.Akad., P. 412 (1841)

FARIA CUNHA, P. 81 (1917)
A. Frg. p. 530 (19æ8)
B. MM. 1. 4. 8. 9. 356. 368. 367. 374.
U. MM. Costa Atlóntica

Coscinodiscus Grani Gough North Sea Fish. Invest., p. 338 (1905)
A. -
B. MM. 1. 2. 3. 4. 6. 351. 363. 364. 365. 368. - R.Grande do Sul PC. p. 32 (1950)
U. MM. Costa Atlántica, Punta del Este, Atlántida

Coscinodiscus he teroporus Ehrenberg Ber.Berl.Akad., p. 265 (1844)
A. -
B. MM. 5. 367. 370.
v. - 
Coscinodiscus Hustedti $i$ Müller-Melchers

com.Bot. Museo Hist. Nat. (1954)

New and little known Diatoms, p. 2, lam. 1, fig. 2, 3, 4, 5

A. - Atlántico sur argentino en la corriente calida del Brasil

B. MM. 5. 7. 334. 364. 365. 369.

U. MM. p. 2(1954)

Coscinodiscus Janischi A.Schmidt

A.Schmidt Atlas, lam. LXIV, fig. 3 \& 4
A. Frg. p. $531(1928)$
B. MM. 365 .
U. MM. Cost.Atl.Ur.

Coscinodiscus Jonesianus (Gréville) Ostenfeld Denkschr.Bot.Archiv, Vol. I1, p. 13 (1915)
A. Frg. p. 536 (1928)
в. MM. 2. 4. 7. 9. 334. 352. 356. 365. 370. 372.
U. MM. Cost.Atl.Ur.

Coscinodiscus lineatus Ehrenberg Abh.Berl.Akad., p. 129 (1838)
A. Frg. p. 530 (19æ8)
MM. p. 321 (1951)
B. MM. 1. 4. 10. 351. 356. 365.
U. MM. Cost. Atl.Ur.

Coscinodiscus nitidus Gregory Trans.Roy.Soc.Edinb., Vol. XXI, p. 27 (1857)

zimmermann, p. 9 (1913) - Rio de Janeiro
A. -
B. MM. 9 .
U. MM. Punta del Este

Coscinodiscus obscurus A. Schmidt

A.S.Atlas, pl. 61, fig. 16 (1878)

ZIMMERMANN , p. 145 (1913) 

A. --
B. MM. 1. 365. 366, 368, 372. 373. 374.
U. MM. Atlántida

Coscinodiscus oculus iridis Ehrenberg Abh.Berl.Akad., p. 147 (1839)

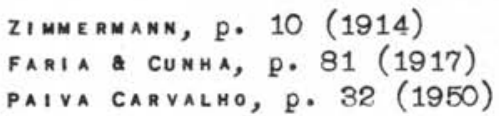
A. Frg. p. $322(1941)$
MM. Atlántico Sur Argentino, Antarctico Argentino
B. MM. 2. 5. 9. 334. 356. 370 .
PC. p. 32 (1950)
U. MM. $\quad 30$ millas al sur de La Paloma, raro en Atlántida

Coscinodiscus Pavillardi Forti

R.Comit.Talasso.|tal., XCVII, p. 124 (1922)
A. MM. Necochea
B. MM. 1. 2. 4. 5. 6. 7. 8.
U. MM. Atlántida, Punta del Este

Coscinodiscus perforatus Ehrenberg Ber.Berl.Akad., p. 78 (1844)
A. MM. Necochea
B. MM. 1. 2. 4. 5. 6. 7. 8.
U. MM. Atlántida, Punta del Este

Coscinodiscus punctiger (Castracane) Müller-Melchers Physis, Vol. XX, no 59 (1953)
A. MM. Atlántico Sur Argentino
B. MM. 1. 2. 5. 7. 8. 356. 363. 363A. 364. 365. 366. 367. 368. 370. 371. 373.
U. MM. (1953)

Coscinodiscus radiatus Ehrenberg Abh.Berl.Akad., p. 148 (1839)

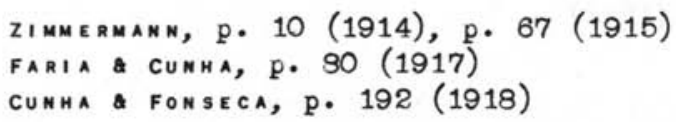



A. Frg. p. 157 (1924)
B. MM. 1. 4. 5. 7. 9. 351. 356. 362. 365. 366. 367. 368. 370. 372. PC. p. $32(1950)$
U. MM. Cost.Atl.Ur.

Coscinodiscus Rothii (Ehrenberg) Grunow

Schneider Nat.Beitr.z.Kennt.d.Kaukasusländer, p. 125 (1878)

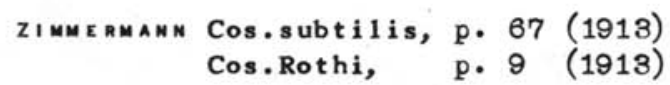
A. Frg. p. $528(1928)$
p. $321(1941)$
B. M. 5. 334. 364. 365. 366. 368. 370 A. 372. 374.
U. MM. Costa Atlántica

Coseinodiscus stellaris Roper

Quart.Jour.Micr.Soc., Vol. VI, p. 21 (1858)
A. MM. Antarctica Argentina
B. MM. 1. 368.
U. MM. Atlántida, Punta del Este

Coscinodiscus variabilis Frenguelli

Diat.oceano At 1., An. Museo Nacional B.Rivadavia, p. 524 (1928)
A. Frg. p. 524 (1928)
B. MM. 5. 368. 370 .
U. MM. Costa Atlántica

\section{Cyclotella Meneghiniana Kützing Bac., p. 50, lam. 30, fig. 68 (1844)}
A. Frg. p. 324 (1941)
B. MM. 5. - raro
U. MM. Atlántida

Cyclotella striata (Kützing) Grunow Kongl.Sv.Vet.Akad. Handl., Vol. 17, p. 119 (1880)

ZI MUERMANM, p. 69 (1913)
A. Frg. p. 330 (1938)
var. ambigua Grunow
var. baltica 


\footnotetext{
V.H.Syn., 1 ám. 92, fig. 12(1881)

V.H.Syn., fig. 13, 14 (1881)

Frenguelli, p. 331 (1938)

Frenguelli, p. 331 (1938)

B. MM. 370. 371 .

U. MM. Atlántida
}

Cyclotella stylorum Brightwell

Quart.Jour.Micr.Soc., Vol. VIII, lam. 6, fig. 16 (1860)

A. Frg. p. 221 (1939)

B. MM. 1. 4. 5. 334. 366. 374. 375.

U. -

"Especie de mares tropicales" (Hustedt)

Cymatosira Adaroi Azpeitia (Lám. I, fig.6; Lám.II, fig. 1 y 2) Diatom. Españolas, p. 201 (1908), lam. IX, fig. 5

Especie fósil en España, lám. IX, fig. 5, en el deposito de 1a Serrata de Lorca: 0,029 $\times 0,007 \mathrm{~mm}$.

La especie brasilera es un poco más grande y las terminaciones más estiradas y agudas; en zona conectival las perlas redondas se distinguen bien: 0,040 a $0,055 \mathrm{~mm}$ de 1 argo $\times 0,009 \mathrm{~mm}$ $6 / 8$ estrias transversales en $0,01 \mathrm{~mm}$ y $1 \mathrm{a}$ misma cantidad de perlas. Frecuente en el Mar de Trapandé.
A. -
B. MM. 1. 4. 5. 334. 351 .
U. -

Dactyliosolen medi terranea $\mathrm{H}$. Peragallo

Diatomiste, vol. I, p. 104 (1892)
A. -
B. MM. 5. 367 .
U. M. Cost.Atl.Ur. Suele tener esporos de resistencia.

Denticula antillarum $\mathrm{Cleve} \&$ Grunow

Diat. West Ind. Arch.

Bih.K.Sv.Akad.Handl., Vol. 5, p. 14 (1878)

ZIMUERMANN, p. 156 (1913)
A. -
B. MM. 351. 354 .
Zim. Santos
v. - 
Diploneis bomboides A.Schmidt

A.S.Nordsee, pl. 1, fig. 2 (1874)

forma minor Cleve, Syn. I, p. 89 (1894)

A. Frg. p. 208 (1939a)

B. MM. 4. 9 .

U. MM. Bstuario Solis Grande y Punta del Este

Diploneis lıtoralis (Donk.) Cleve Donkin., B. II, p. 5, Pl. I, fig. 2 (1870)

Cleve, Syn. I, p. 94 (1894)

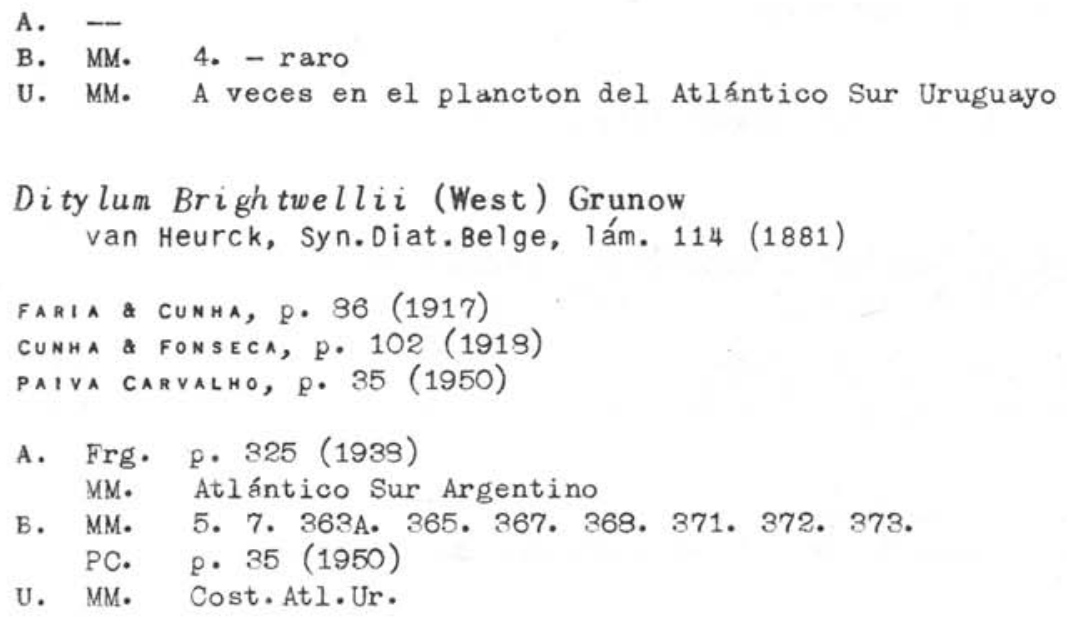


Eucampia zoodiacus Ehrenberg

Ber.Berl.Akad., p. 151 (1839)

FARIA A CUNHA, P. 84 (1917)
A. -
B. MM. 365 .
U. MM. Costa Atlántica

Eupodiscus antiquus Cox (Lám. II, fig. 3)

Kain \& Schultze, Bull.Torrey Bot.Club, Vol. 16, p. 209 (1889)

ZI MMERMANN, P. $143(1913)$
A. --
B. MM. 1. 2. - Rio Guama, Belem
U. -

Guinardia flaccida (Castracane) Peragallo

Diatomiste, Vol. I, p. 107 (1892)

FARIA \& CUNHA, p. 80 (1917)

Cunha Fonseca, p. 102 (1918)
A. --
B. MM. 1. 2. 8. 9.
U. MM. Costa Atlántica, en invierno más amenudo

Gyrosigma balticum (Ehrenberg) Rabenhorst Abh.Berl.Akad., p. 114 (1830)

Rabenhorst süssw. Diat., p. 47 (1853)
A. Frg. p. $291(1938 \mathrm{a})$
B. MM. 1. 4.5 .
U. MM. Punta del Este

Gyrosigma fascicola (Ehr.) Griff. et Henfr. Micr.Diet., lam. II, fig. 20 (1875)
A. Frg. p. 294 (1939)
B. MM. 7. - raro
U. MM. Punta del Este

Gyrosigma strigile W. Smith

syn.Brit.Diat., p. 208 (1852) 


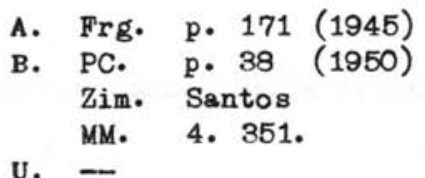

Hemidiscus cuneiformis Wallich var. ventricosa Hustedt Trans.Micr.Soc., Vol. 8 (1860)

FARIA Q CUNHA, p. 81 (1917) - oceanico - tropical \& subtropical
A. --
B. MM. 4. 9. 352. 354. 356. 357. 365. 366.
Faria \& Cunha, oceanico, frecuente
U. --
Hemidiscus ovalis Lohmann (Lám. II, fig. 4) Pliocen Diatoms from the Kettleman Hills (California)
Geol.Surv. (1938) Prof.Paper no $189 \mathrm{C}$
A. -
B. MM. 1. 5. 8. 351. 365. 369.
U. -

Valva ovalada, forma cuneata. Areolas pequeñas perlas 10/12 en .01mm. Centro un espacio ovalado bordeado por una fila de perlas ligeramente más grandes alrededor de una perla mayor. Borde 16 estrias en .01mm. Largo 0,03X0,02mm. No tiene ocello.

Frecuente en Mar de Trapandé. Fn el Atlántico desde San Francisco hasta Ilheos. Fósil California.

Las dos otras especies muy parecidas son:

Euodia margaritacea Brun. (Diat.fos. Japón)

lám. IV, fig. 6, a, b, pero el tamaño es mucho mayor. $0,070 \times 0,050$. Brun. agrega, p. 37: "aussi trouvé dans un sondage $(5307 \mathrm{~m})$ de la mer d'Arafura. Castracane."

Euodia sp. en Karsten "Valdivia" Exp.Antarctica, p. 122, lám. XVII, fig. 6, a $4605 \mathrm{~m}$ de profundidad.

Es de suponer que todas estas formas están muy cerca a H.ovalis. Interesante es que se encontró en el Atlántico Sur $54^{\circ} 46^{\prime} \mathrm{S}-26^{\circ} 39^{\prime} \mathrm{W}$ (Karsten, p. 49).

Hemiaulus membranaceum Cleve Bih.K.Sv.Akad.Hand1., Vol. I, n이 II, P. 6 (1873) 
A. -
B. MM. 368. 371. 375. - probablemente oceanico (Allen \& Cupp p. 170)
U. -

Hemiaulus sinensis Gréville Am. Nat.Hist., Vol. 16 (1865)

FARIA CUNHA, p. 84 (1917)

müller-melchers, p. 8 (1954), lám. Vit, fig. 4, 5.
A. --
B. MM. 5. 6. 7. 3. 365. 368.
U. MM. Punta del Este, Atlántida

Hemidiscus Hardmanianus Gréville Descr. New and Rare Diat. (1865)

Allen Cupp, Java Sea, p. 152 (1935)
A. -
B. MM.
2. 4. 9. 356. - relativamente frecuente
U. -

Hyalodiscus laevis Ehrenberg Ber.Berl.Akad., p. 70 (1845)

Microgeol., lam. 33, fig. 15, 17 (1854)
A. -
B. MM. 356 .
U. -

Hyalodiscus scoticus (Kütz.) Grunow Jour.Roy.Micr.SOC., p. 690 (1879)
A. Frg. p. 297 (1930)
MM. p. $322(1951)$
B. MM. 8.7 .
U. MM. Punta del Este, Atlántida

Is thmia enervis Ehrenberg Infus., p. 209, lam. 16 (1838)

FARIA a CUNAA, Ist.ob1iquata, p. 85 (1917)

A. - 


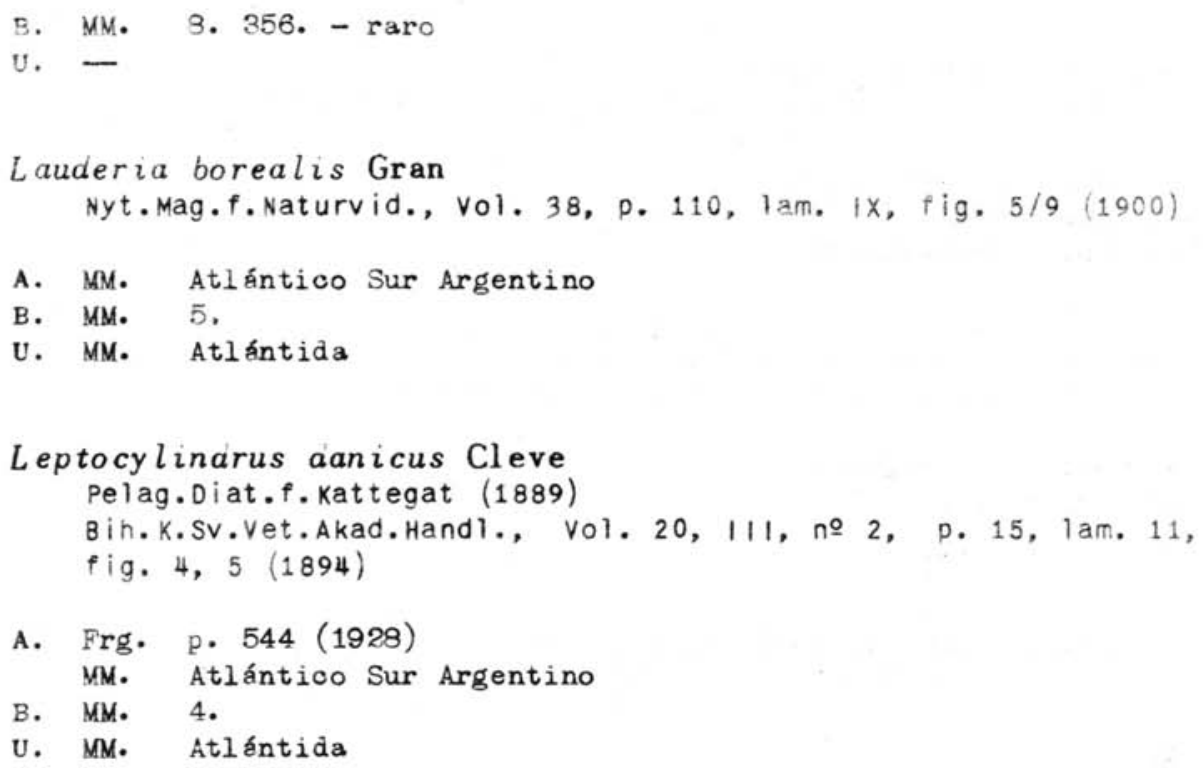

$L i$ thodesmium undulatum Ehrenberg

Abh.Berl.Akad., p. 75 (1840)

FARIA a CUNHA, p. 86 (1917)

Cunha Fonseca, p. 102 (1918)
A. Frg. p. 520 (1928)
MN. p. 322 (1951) - Atlántico Sur Argentino
B. MM. 1. 2. 5 .
U. MM. Atlántida

Melosira granulata (Ehrb.) Ralfs

Pritchard Infus., p. 820 (1861)
A. Frg. p. 136 (1935)
B. MM. 365. 370. 373. - R. Grande do Sul, barra. Rio Guama.
U. MM. Atlántida

Melosira setosa Gréville

Diat.Tropics, p. 436, lam. 6, fig. 17/19 (1866)
A. Frg. p. $331(1938 a)$
MM. Necochea
B. MM. 1. 3. 4.
U. MM. Atlántida, muy raro 
Navicula brevis Gregory

Trans.Roy.Soc.Edinb.Diat.Clyde, 21, p. 6 (1857)

A. Frg. p. 152 (1925)

B. MM. 371 .

U. MM. Cost.Atl.Ur.

Navicula cancellata Donkin (1861)

Cleve \& Grunow, Arkt.Diat., p. 36 (1880)
A. MM. p. $322(1951)$
B. MM. 351.
U. -

Navicula humerosa Brébisson (1856)

W.Smith, Br.Diat., II, p. 93
A. -
B. MM. 378.
U. MM. Atlántida, Punta del Este

Navicula lyra Ehrenberg (Lám. II, fig. 6) Abh. Akad.Berlin (1843)

A. Frg. p. $216(1939 b)$

B. MM. 9. - raro en plancton

U. MM. Punta del Este, muy raro en plancton

Llamo la atención a esta especie por la forma de los rayos de la lira, que son completamente paralelos; solamente en un lado de la valva existe una pequeña deviación hacia el centro. Las típicas formas tienen la forma de la lira bien definida. También en el tipo del Uruguay las partes terminales de la lira son rectas y casi paralelas.

Navicula marina Ralfs

Donk.B.D., P. $19(1858)$
A. --
B. MM. 5 .
U. MM. Atlántida, raro

Navicula pennata A. Schmidt A.S.Atlas, pl. 1, fig. 11 

A. Frg. p. 256 (1930)
B. MM. 334. 367 .
U. MM. Atlántida

Navicula scopulorum Brébisson Kützing, Spec.Alg., p. 81 (1849)
A. Frg. p. 287 (1938)
B. MM. 5.7 .8 .
U. -

Nitzschia circumsuta (Bailey) Grunow Arkt ische Diat., p. 77 (1880)

ZI MUERMANM, p. 47 (1913)
A. -
B. MM. 370 .
U. -

Nitzschia granulata Grunow Arkt.Diat., p. 68 (1880)

ZIMUERMANM, p. 131 (1913)
A. Frg. p. 68 (1926)
B. MM. 1 .
U. MM. Barra Solis Chico

Nitzschia littoralis Grunow Arkt.Diat., p. 75 (1880)

ZIMUERMANN, p. 46 (1913)
A. -
B. MM. 9 .
U. -

Nitzschia oxyrhynchus Frenguelli

Physis, Diat. Durazno \& Brusquitas, p. 170 (1925)
A. Frg. p. 170 (1925)
B. MM. 371 .
U. MM. Barra Solis Chico 


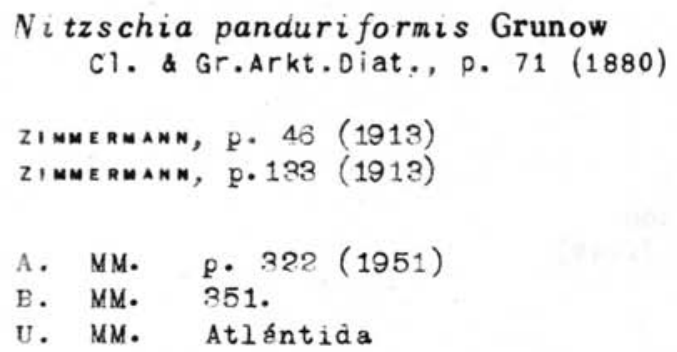

Nitzschia scalaris (Ehrenberg) Wm. Smith synopsis, 1, p. 39, lám. 14, fig. 115
A. Frg. p. 290 (1941)
B. MM. 334. - fragmentos
U. MM. en turba subfosil (1945)

Nitzschia seriata Cleve Vega Exped., Vol. 3, p. 478 (1883)

FARIA \& CUNHA, P. 86 (1917)
A. MM. Atlántico Sur Argentino
B. MM. 365. 368 .
PC. p. 38 (1950)
U. MM. Atlántida

Nitzschia (Nitzschiella) ventricosa (Palmer) Kitton Kitton, New Spec., p. 206, lám. 38, fig. 5 (1873)

Frenguelli, Nitz,ventricosa (1945)
A. -
B. MM. 9 .
U. Frg. La Paloma
MM. La Paloma, Punta del Este

Nitzschia virgata (Roper) Grunow

C1. \& Grunow, Arkt.Diat., p. 104 (1880)
A. Frg. p. 176 (1925)
B. MM. 371 .
U. MM. Atlántida 
Nitzschia tryblionel la Hantzsch

cl. \& Grunow, Arkt.Diat., p. 69
A. Frg. p. 68 (1926)
в. MM. 5. 369 .
U. -

Nitzschia paradoxa (Gmelin) Grunow

Grunow in van Heurck Syn., p. 176 (1880-85)

FARIA CUNKA, P. 86 (1917)

cunha a fonseca, p. 103 (1918)
A. Frg. p. 199 (1945)
B. M. 1. 7. 334 .
U. MM. Atlántida

Opephora Martyi Héribaud

Diat.Foss. d'Auvergne, p. 43 (1902)
A. Frg. p. 309 (1941)
B. MM. 9 .
U. MM. Atlántida
Paralia sulcata Cleve
Arctic Sea, p. 7 (1873)
ZIMUERMANN, p. 124 (1914), p. 69 (1915)
FARIA CUNHA, P. 80 (1917)
Cumha FonsecA, p. 10 (1918)
A. Frg. p. $153(1924)$
в. MM. 1. 2. 5. 7. 9. 334. 367. 369. 370. PC. p. 31 (1950)
U. MM. Atlántida
Plagiogramma antillarum $\mathrm{Cleve}$ Diat. West ind.Arch.$$
\text { Bih.K.Sv.Akad.Handl., Vol. 5, p. } 10 \text { (1878) }
$$
A. -
B. MM. 351. - raro
U. -- 
Planktoniella (Wallich) Schütt

pflanzenleben der Hochsee, p. 20 (1893)
A. -
B. MM. 334. - la valva central chica - 0,02365 - deja duda si
v. - se trata de esta especie.
Pleurosigma affine Grunow
Cl. \& Gr., Arkt.Diat., p. 51 (1880)
A. Frg. p. 296 (1938a)
MM. Necochea (1951)
B. MM. 354. 365. 368. 370 .
U. MM. Cost.Atl.Ur.

Pleurosigma naviculaceum Brébisson (1854) Cherbourgh, p. 17, fig. 7
A. Frg. p. 260 (1930)
B. M. 4. 5. 9. 352 .
U. -

Pleurosigma Normanii Ralfs

pritchard Infus., p. 919 (1842)
A. Frg. p. 507 (1928)
B. MM. 4. 5 .
v. -

\section{Pleurosigma acutum Norman} Norman en Pritchard, Infus., p. 920 (1861)

Cleve, synopsis I, Vol. 26, p. 42 (1894)

La especie fué determinada por gentileza del Dr. J. Frenguelli, de La Plata, como no coincidia con las formas conocidas. Es más grande que la de Peragallo - Mer de France y más ancho que las descritas por Cleve (1894). Doy una nueva definición sobre la del material brasilero.

Pleurosigma acutum f. brasiliana Müller-Melchers

Valva lanceolada, sigmoide. Terminación de la valva notablemente aguda. 0,262 a 0,305m largo por 0,032 a 0,038 de ancho. 
Estrias muy finas, difícil de definir. Línea mediana sigmoide, excéntrica. En la última parte toca al borde. Nódulo pequeño, redondo.
A. -
B. MM.
5. - bastante frecuente
U. -

Podosira maxima (Kütz.) Grunow

$$
\text { Cl. \& Gr., Arkt.Diat., p. } 118 \text { (1880) }
$$

A. Frg. p. $196(1939 b)$

MM. Necochea

MM. p. $322(1951)$

B. MM. 5. 7. 8 .

U. MM. Punta del Este, La Paloma

Podosira stelliger (Bailey) Mann

Diat. Albatross, p. 242 (1907)

Zi MUERMAN N, p. 70 (1913), p. 146 (1913)
A. Frg. p. 540 (19æ8)
MM. $38^{\circ} 32^{\prime} \mathrm{S}-57^{\circ} 08^{\prime} \mathrm{W}$
B. M. 1. 9 .
U. MM. Atlántida, Punta del Este

Polymyxus coronalis Bailey

$$
\text { I.W.Bailey, Jour.Nat.Hist., p. } 341,1861
$$

ZI MMERMAMN, p. 11 (1913)
A. -
B. MM. Rio Guama
U. -

Pseudoeunotia doliolus (Wallich) Grunow

$$
\text { V.H.Syn., pl. } 35 \text { (1880) }
$$
A. -
B. MM. 351. 365. 367 .
U. -

Raphoneis amphiceros Ehrenberg Ber.Berl.Akad., p. 87 (1844) 
Z I MUERMANN, p. 50 (1913), p. 137 (1913)
A. Frg. p. 279 (1924)
MM. p. 322 (1951)
B. MM. 368. 375 .
U. MM. Atlántida

Raphoneis Castracanei Grunow

V.H.Syn., lám. 36, fig. 28 (1881)
A. Frg. p. $309(1938 b)$
B. MM. 5. 7. - rara
Zim. Itaparica
U. -

Raphoneis surirella (Ehr.) Grunow V.H.Syn., lám. 36 (1880)
A. Frg. p. 509 (1928)
B. MM. 5. 9. 359 .
U. MM. Cost. Atl.Ur.

Rhabdonema adriaticum Kützing Bacill., P. 126 (1844)

FARIA \& CUNA, p. 86 (1917)
A. Frg. p. 290 (1930)
B. M. 9. 352. 356.
U. MM. Atlántida

Rhizosolenia alata Brightwell Quart.Jour.Micr.Soc., Vol. VI, p. 96 (1858)

FARIA CUMHA, p. 82 (1917)

cuma fonseca, p. 102 (1918)

f. gracilima (Cleve) Grunow V.H.Syn., lám. 79 (1881)

cunha a fonseca, p. 102 (1918)
A. MM. Atlántico Sur Argentino
B. MN. 1. 2. 4. 5. 364. 365.
U. MM. Cost.Atl.Ur. 
Rhizosolenia alata f. indica (Perag.) Ostenfeld vid.Medd.Nat.For. Kbhvn. (1901)
A. Frg. p. 560 (1928)
B. MM. 368. 1. 2. 4.5.
U. MM. Atlántida

Rhizosolenia Bergoni H.Peragallo

Diatomiste, vol. I, p. 110 (1892)
A. -..
B. MM. 5 .
U. Ma. Atlóntida
Rhizosolenia calcar avis M. Schultze Müllers Archiv, p. 339 (1858)
FARIA CUNHA, p. 82 (1917)
cumba fonseca, p. 102 (1918)
A. Frg. p. 558
MM. Atlántico Sur Argentino
B. MM. 2. 4. 8. 368.370 .376$.
U. MM. Cost.Atl.Ur. - especialmente sobre la plataforma atlán- tica (oceanica!).

Rhizosolenia imbricata Brightwell

$$
\text { Quart.Jour.Micr.Soc., vol. VI (1858) }
$$

A. Frg. p. 558 (1928)

Mu. Costa Atlántica Argentina

B. MM. 5. 365. 367. 368. 372. 373. 374. 375.

U. MM. Cost. Atl. Ur.

var. Shrubsolei (Cleve) Schröder

vierteljahrschrift Nat.Ges. Zürich, vol. 51 (1906)

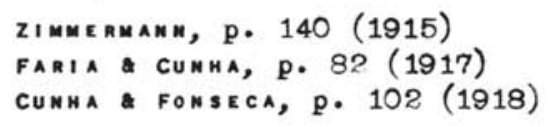
A. Frg. p. 558 (19æ8)
MM. Atl f́ntico Sur Argentino
B. MM. 4. 368 .
PC. p. 33 (1950)
U. MM. Cost.Atl.Ur. 
Rhizosolenia robusta Norman

Pritchard Infus., p. 866 (1861)

FARIA CUMAA, p. 82 (1917)
A. Frg. p. $556(1928)$
MM. Atlántico Sur Argentino
B. MM. 2. 4.365 .368 .
PC. p. 34 (1951) Rhisosolenia sp., fig. 1 ?
U. MM. Atléntida, raro

Rhizosolenia setigera Brightwell

Quart.Jour.Micr.Soc.,"p. 95 (1858)

FARIA CUMHA, p. 82 (1917)

FREMUUELL I, p. 557 (1928)

zimuramann, Rio de Janeiro
A. Frg. p. 557 (19:8)
MM. Atlántico Sur Argentino
B. MM. 1. 2. 3. 4. 5. 7. 329. 367.
U. MM. Atlántida, raro

Roperia tessellata (Roper) Grunow

V.H.syn., lám. 118 (1881)

A. -
B. M. 334.
U. MM. Punta del Este, La Paloma, Sta.Teresa

Sceletonema costatum (Gréville) Cleve (Lám. II, fig. 1-B)

Trans.Micr.Soc.N.S., Vol. 14, p. 77 (1866)

Cleve Bih.K.Sv.Vet.Akad.Handl., Vol. V, p. 18

FARIA CUNHA, p. 80 (1917)
A. Frg. p. 541 (19æ8)
B. MM. 5. 10. 365. 373 .
U. MM. Atlántida, Punta del Este

Schizostauron crucicula Grunow

cleve, New Diat., p. 16 (1881)

Z I MUE R A AN W, p. 41 (1913) 


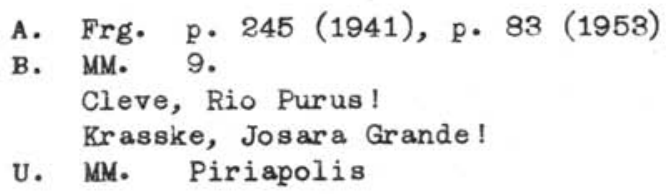

Stepnanopyxis Palmerianum (Gréville) Grunow

Trans.Micr.SCC.N.S., Vol. XIII, pl. 1 (1865)

Grunow, Denk.kais.Ak.wiss., Vol. 48, p. 90 (1884)
A. -
B. MM. 2. 4. 364. 368. 372.
U. MM. especie de aguas cálidas del Brasil. Atlántida, rara

Stephanopyxis turris (Gréville and Arnott) Ralfs

Trans.Ruy.Soc.Edinb., Vol. 21, p. 64 (1857)

Ralfs en Pritchard infus., p. 826 (1861)

ZIMUERMAMN, P. 5 (1914)

FARIA CUNHA, p. 80 (1917) St,appendiculata
A. Frg. p. 538 (1928)
MM. Atléntico Sur Argentino
B. MM. 3. 4. 5. 7. 364. 365. 370.
PC. p. $33(1950)$
U. MM. Cost.Atl.Ur.

Surirella fastuosa Ehrenberg

w.Smith, Brit.Diat., p. 32 (1852)
A. Frg. p. $191(1939 b)$
B. MM. 351.356 .
U. -

Surirella Febigerii Lewis (Lám. II, fig. 5)

A.S.Atlas, pl. 20, fig. 9 (1885) (San Frsco.Calif. Janisch.)
A. -
B. MM. 1. 8. 349. - entre Santos y Rio de Janeiro
MM. Rio Guama, raro
Er. Schmidt Atlas, lám. 21 se describe Sur.praeclara
U. - Weisflog, que es muy parecicia (Santos). 
Surirella gemma Ehrenberg

kreide Form., p. 76 (1839)

ZI MUERMAMN, P. 94 (1916)

Paiva carvalho, p. 89 (1950)

A. Frg. p. $312(1938$ a)

B. M. 1. 4. 5. 6. 8. - Barra Rio Grande do Sul

PC. p. $39(1950)$

U. -

Surirella rorata Frenguelli

Diat. de la Mar Chiqu. Inst. Museo La Plata, p. 131 (1935)

QUERAMDIMEMSE, P. 295 (1938a)

KRASsKE, Kies. Brasil I, p. 559 (1939)

A. Frg. p. 131 (1935)

B. Mi. 9 .

MM. Rio Grande do Sul - barra

U. MM. Arroyo Solis Chico, en aguas salobres

Surirella striatula Turpin

Genus surirella, p. 3 (1828)

ZIUMERMAMN, p. 48 (1913)

A. Frg. p. 146 (1924)

B. M. 371.

U. MM. Punta del Este

Synedra crystallina (Ag.) Kützing Bacill., p. 69 (1844)

A. -

B. M. 9.

v. -

Synedra ulna (Ni tzsch. )Ehren berg var. amphirhynchus (Ehr. )Grunow(1838) verh.Zool.Bot.Ges.Wien, vol. 12, p. 397 (1862)

A. Frg. p. 166 (1925)

B. M. 9 .

U. Mu. Atlántida 
Synedra undulata (Bailey) W. Smith Bailey Smithsonian Contrib., Vol. 7, p. 15

Toxarium undulatum (1853)

W. SMITH SYN., II, p. 97 (1856)

A. Frg. p. 217, II, fig. 24 (1939)

B. MM. 9. - fragmentos

U. -

Trachyneis aspera (Ehrenberg) Cleve synopsis, I, p. 191 (1894)

A. Frg. p. 257 (1930)

B. MM. 5. 9. 334 .

U. MM. raro, Atlántida, La Paloma

Trachyne is aspera var. oblonga Bailey Smith.contrib., p. 10, fig. 17 (1854)

A. --

B. MM. 356.

U. -

Terpsinoe americana (Bailey) Ralfs New species, p. 7 (1854)

Pritchard Infus., p. 859 (1861)

ZIMMERMANN, p. 65 (1915)

Paiva carvalho, p. 35 (1950)

A. Frg. p. $322(1938 \mathrm{a})$

B. MM. 1 .

p. 316 (1941)

PC. p. 35 (1951)

Bailey, W., Pará River (1862)

MM. Rio Grande do Sul (barra)

U. -

Terpsinoe musica Ehrenberg Abh.Berl.Akad., p. 402 (1841)

Zi MuERMann, p. 141 (1913)

A. Frg. p. 200 (1945) 
B. MM. Rio Grande do Sul - barra

U. MM. Arroyo Solis Chico

Thalassionena nitzschioides Grunow

V.H.Syn., p. 43 (1881)

FARIA \& CUNHA, P. 86 (1917)

CUnha a Fonseca, p. 102 (1918)

HENDEY, P. 336 (1937)

A. Frg. p. 511 (19æ8)

MM. Atlántico Sur Argentino

B. MM. 1. 2. 4. 5. 6. 8. 365. 367. 370. 372. 374.

PC. p. 36 (1950)

u. MM. Cost. Atl.Ur.

Thalassiosira decipiens (Grunow) Jørgensen

Schneider Nat.Beitr. Kennt.Kaukasus., p. 125 (1878)

Jørgensen Hydr.Biol. Invest. Norw.Fjords, p. 96 (1905)

A. Frg. p. 328 (1938a)

B. MM. 363A. - sueltos, no en cadenas

U. MM. En el plancton, sueltos, no en cadenas, raro. Algunas formas pequeñas de Cos.excentricus son dudosas y pueden pertenecer aqui 6 a P1anktonie11a.

Thalassiosira subtilis (Ostenfeld) Gran

Jagttag. Ov.Temp.Salt.Plankt., p. 55 (1899)

Gran Nyt.Mag.Naturv., Vol. 38 (1900)
A. -
B. MM. 5 .
U. MM. Atlántida

Thalassiothrix Frauenfeldi $i$ Grunow

K.Sv.Vet.Akad.Hand 1., Vol. 17, P. 109 (1880)

Z I MUERMANN, p. 159 (1913)
A. -
B. MM. 365. 366. 369 .
PC. p. $36(1950)$
U. - 
Thalassithrix mediterraneum Pavillard

Diat.Pelag. du Golfe de Lyon, p. 39 (1916)
A. --
B. MM. 7 .
U. MM. Cost.Atl.Ur.

Triceratium alternans Bailey

Smithson. Contr. know1., Vol. 2, p. 14 (1851)
A. Frg. p. $517(1928)$
MM. p. $323(1951)$
B. MM. 2. 251 .
U. MM. Cost.Atl.Ur.

Triceratium favus Ehrenberg Abh.Berl.Akad., p. 159 (1839)

ZIMMERMANN, p. 65 (1913), p. 141 (1913)
A. Frg. p. 317 (1941)
B. MM. 1. 2. 4. 9. 235. 365. 367. - Rio Guama
MM. 367. 352. - forma quadrangularis (Lám. II, fig. 8)
U. MM. excepcional

Trıceratıum formosum Brightwell (Lám. II, fig. 9) Quart.Jour.Micr.Soc., Vol. 4, p. 274 (1856)

f. quinquelobata Gréville Trans.Micr.Soc.London N.S., Vol. 14, p. 83 (1866)
A. --
B. MM.
U. -

Trıceratium pe,tuc inus (Ehr.) Wallich (Lám. II, fig. 7) Quart.Jour.Micr.Soc., vol. 6, p. 249 (1858)

ZI MMERMANN, p. 141 (1913)
A. -
B. MM.
U. - -
9. - muy raro 
Triceratium scitulum Brightwell

Triceratium, Quart.Jour.Micr.Soc., p. 250 (1853)

ZIMMERMANM, p. 142 (1913)

FRENGUELLI, p. 155 (1924)

A. Frg. p. $155(1924)$
MM. p. $323 .(1951)$
B. MM. 2. 365.
MM. Rio Guama, Belem
MM. Rio Grande do Sul, barra
U. MM. Cost. Atlitr.

De las muestras se determinaron 182 diatomeas para el Brasil, de las cuales se encuentran también 126 en el Uruguay y 114 en la Argentina. No todas son planctonicas, hay tipos neríticos y de agua salobre. En algunos casos hay especies de agua dulce, ejemplares perdidos que han sido llevados al mar y aparecieron así en el plancton. Las muestras de la motonave "Sta.Catarina" contienen también una cantidad de especies neríticas, esto se demuestra cuando la nave se aproxima a la costa, como en las muestras cerca de Santos, Rio de Janeiro, etc. Así se demuestra el desplazamiento del material nerítico hacia la mar. Por ejemplo Hemidiscus ovalis se encuentra también en la mar, como en el caso de muestras de San Francisco do Sul. De Rio de Janeiro para el Norte encontramos Hemidiscus membranaceus. Es especie tropical, como también Asterolampra marylandica. Para el Sur, en el Uruguay se suele encontrar durante el invierno Chaetoceros coarctatus - especie comun en las muestras brasileras. Aparece de repente en las costas uruguayas para desaparecer en seguida. Habrá sido desplazado por la corriente cálida del Brasil. Esto son unas pocas incógnitas que se presentan en el estudio del plancton y que habrá de estudiar más a fondo. No es difícil de pescar de abordo de una nave por medio de la bomba de agua de mar. Habrá que usar redes más grandes que la que entregué al Ing. Weiher que no tenia más de 120 centimetros cuadrados de superficie filtrante. El material era de Nylon. De este modo los gastos de conseguir muestras no serán grandes.

Repito una vez más. Este estudio ha sido compaginado para guia en investigaciones futuras y no pretende ser completo. Desde las costas del Brasil habrá que empezar un estudio sistemático del plancton - no solamente de las diatomeas sino de todo el micro ambiente marino. 


\section{RES UMO}

Versa o presente trabalho sôbre Diatomáceas do plancton marinho dos Estados de S.Paulo e R.G. do Sul, além de espécimes colhidos na costa bram sileira até os rochedos S.Pedro e S.Paulo, pelo Eng. J. Neiner, durante a viagem por êle feita a Europa.

Baseou-se o autor, pois, em três coleções das quais logrou determinar 192 Diatomáceas parao Erasil e em que se encontravam também 126 peculiares as costas uruguaias e 114 frequentadoras das águas argentinas. Foi feita a comparacâo entre o material brasileiro e o das águas do Uruguai e Argentina.

Assinala o autor que nem tôdas sêo planctônicas, havendo no acervo colhido, representantes neriticos e de água doce. Dentre êstes existem espécimes desgarrados que foram parar no plancton marinho.

Dentre o material colhido pelo' Eng. Weiher figuram também espécies neriticas colhidas mais próximo da costa, sobretudo a se aproximar o navio de Santos e R. de Janeiro. Comprova-se assim o deslocamento do material neritico, rumo ao mar aberto. Exemplo disso temos em Hemidiscus ovalis, encontrado também no mar largo, proveniente das amostras de S.Francisco do Sul. Do R. de Janeiro para o Norte encontrou o autor Hemidiscus membranaceus e Asterolampra marylandica, ambas tipicamente tropicais. Para o sul, no Uruguai, durante 0 inverno, encontra-se, as vêzes, Chaetoceros coarctatus, espécie frequente nas amostras brasileiras, que ló surge repentinamente para desaparecer logo a seguir. Julga - autor que se trate de representante desviado pela corrente equatorial do Brasil, fazendo ver que existem incognitas que se apresentam a quem se entrega a estudo do plancton e que precisam ser resolvidas mediante estudo de maior profundidade.

Finalizando, lembra o autor que o presente trabalho foi efetuado com o objetivo de servir de guia a futuras investigąões, nâo tendo, portanto, a pretençâo de ser completo. Julga ainda que há necessidade de se estudar sistematicamente $\circ$ plancton da costa brasileira, compreendendo nêsse estudo não só as Diatomáceas, mas todos os micro-organismos existentes no ambiente marinho.

\section{S U M MA R Y}

This paper deals with the Diatomacea from the plankton collected at the coast of the States of Sáo Paulo and Rio Grande do Sul (Brazil), as well as with some specimens gathered along the brazilian coast (up to S.Pedro and S.Paulo Rocks), by Eng. J.Weiner, during his voyage to Europe.

Three collections were exallined from which 182 Diatomacea were determined as occurring in the Brazilian coast, as many as 126 of them being also peculiar to Uruguay coast while 114 were recorded from Argentine waters.

The material from Erazil was compared with that from the coasts of Uruguay and Argentine. 
The author points out that not all of them are planktonic species since the material collected contained neritic as well as fresh water specimens. Among them there were strayed individuals that reached the marine plankton. Neritic species collected nearer the coast were also present in the material collected by Eng. Weiher, specially when the vessel approached Santos and Rio de Janeiro. This fact confirms the displacement of neritic material towards the open sea. For instance, Hemi discus ovalis was also found in the open sea, in off shore samples from S. Francisco do Sul. Hemidiscus membranaceus and Asterolampra marylandica, both typically tropical species were found from Rio de Janeiro northwards. Chaetoceros coarctatus, frequent species in the Brazilian samples may be found in Uruguay during the winter months, when it appears all of a sudden and soon disappears. According to the author, it might well be an individual carried by the Erazilian Equatorial Current. These are, therefore, doubts that occur to all who devote themselves to the study of plankton and further investigations are necessary in order to find the answer to these questions.

As a conclusion the author reminds that this paper was written with the purpose of serving as a guide for future investigations and it is far from being complete. Attention is called to the fact that it is necessary to carry on with systematic studies with the plankton from the Brazilian coast, including not only the Diatomacea but also all the micro-organisms that live in the marine environment.

\section{B I B L I O G R A F I A}

ALLEN, W.E. \& CUPP, E.E.

1935. Plancton Diat. Java. Ann.Jard.Bot. Buit., Vol. XLIV.

CASTRACANE, F.

1896. Rep. on Diat. Challenger.

CLEVE, P.T.

1878. Diat. West Ind.Archip. K.Sv.Vet.Ak.Hdlg., Vol. 5. Stockholm.

1894. Syn.Nav.Diat. K.Sv.Vet.Ak.Hdlg., Vol. 26/Z7. Stookholm.

1900. Plant.f.South Atlantic Ofvers. K.Sv.Vet. Ak.Hdlg. Stockholm.

CLEVE, P.T. \& GRUNOW, A.

1890. Arkt. K.So.Vet. Ak.Halg., Vol. 17.

CUPP, E.E.

1943. Mar.Plankt. Diat. West Coast. Univ.Calif., Vol. 5, no 1. 
CUNHA, A. \& FONSECA, 0 .

1918. Micropl. Costas Mer. Brasil. Osw.Cruz, Vol. X.

FRENGUELLI, J.

1923. Diat.Arr.Brusqu. \& Durazno. Physis, Vol. VIII.

1924. Diat. Tierra Fuego. Univ.Nac. Buenos Aires.

19æ8. Diat. Oceano Atl. Museo Nac.Hist.Nat.

1930. Diat. Costa Atlant. Museo Nac.Hist. Nat.

1931. Diat. Bahia Sanguinetti. Soc.Ctfca. Sta. Fé.

1935. Diat. Mar Chiqu. Museo La Plata.

1938a. Diat. Querandin. Univ.Nac.La Plata.

1938b. Diat. Bahia San Blas. Univ. Nac.La Plata.

193a. Diat. Rada Tilly. Univ.Nac. La Plata.

1939b. Diat. Golfo San Matias. Univ.Nac.La Plata.

1941. Diat. Rio de La Plata. Univ.Nac.La Plata.

1945. Diat. del Platense. Univ.Nac.La Plata.

1953. Diat. Terr. Misiones. Univ. Nac.La Plata.

GOMES DE FARIA, J. \& CUNHA, A. MARQUES DA

1917. Micropl. Baia Rio de Janeiro. Osw.Cruz, T. IX, 1.

GRUNOW, A.

1884. Diat. v. Franz Jos.Land. Denkschr. 48. Wien.

GRAN, H.H.

1905. Nord. Plancton. Jena.

HENDEY, N.J.

1937. Plant.Diat.South Seas. Di scov.Rep., Vol. XVI.

HUSTEDT, Fr.

1927-28. Die Kieselalgen.

KARSTEN, G.

1905. Deut. Tiefsee Exp. Valdivia Arkt. Meere.

1906. Deut. Tiefsee Exp. Valdivia Atlant.Ocean. 
KRASKE, G.

1939. Kieselalgen Brasil I. Arch.f.Hydrobiologie, Vol. XXXV.

LOHMANN, $\mathrm{H}$.

1912. Beitr.Fahrt. Deutschland Atlant.Ocean.

LOHMAN, K.E.

1938. Diat. Kettleman Hills. Geol.Surv., 189-C.

MANN, A.

1907. Diat. Albatross Voyag. U.S.Nat.Herb., Vol. 10.

MÜLLER-MELCHERS, F.C.

1945. Diatomeas de turbas del Uruguay. Com.Bot., Vol. 1, $n \circ 17$.

1949. Chaetoceros de Atlántida. Lilloa, Vol. XIX. Tucuman.

1951. Actinopt.Frenguellii. Physis, Vol. XX, nㅇ 58. B. Aires.

1952. Bid.chin. Com.Bot., n 26. Montevideo.

1953. Diat.Planct. Physis, Vol. XX, n으 59. B.Aires.

1954. New \& Little Known Diat. Com.Bot. n으 30. Montevideo.

PAIVA CARVALHO, J. de

1950. O Plancton doRio M.Rodrigues. Bol.Inst.Paul. Ocean., Vol.I,F.1.

PERAGALLO, H. \& $\mathrm{M}$.

1897. Diat. Mar. de France.

ZIMMERMANN, C. S.J.

1914-16. Diatomaceas E.U. do Brasil. Brotéria, Ser.Botanica.

殅

Lámina 1

Fig. 1 - Asteromphalus Hookerii Ehrenbera

Fig. 2 - Campylodiscus Daemelianus Grunow

Fig. 3 - Campylodiscus Daemelianus Grunow

Fig. 4 - Chaetoceros affinis Lauder

Fig. 5 - Coscinodiscus brasiliensis n.sp.

Fig. 6 - Cymatosira Adaroi Azpeitia (de costado. en cadenas)

Fig. 7 - Biddulphia antediluviana var. excavata Frenguelli

Fig. 8 - Coscinodiscus divisus Grunow 

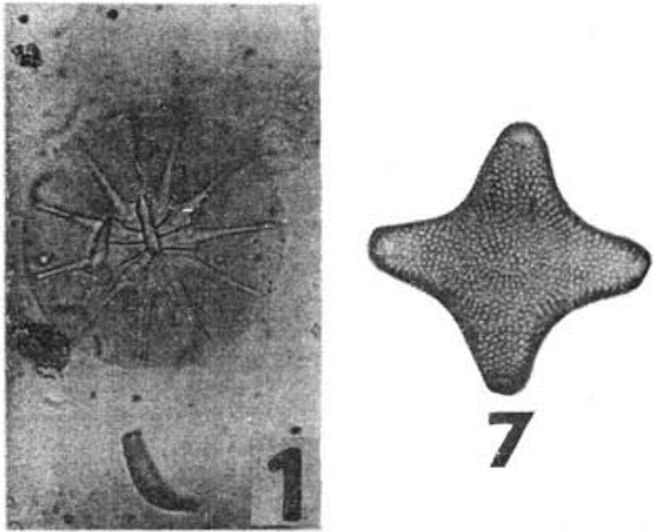
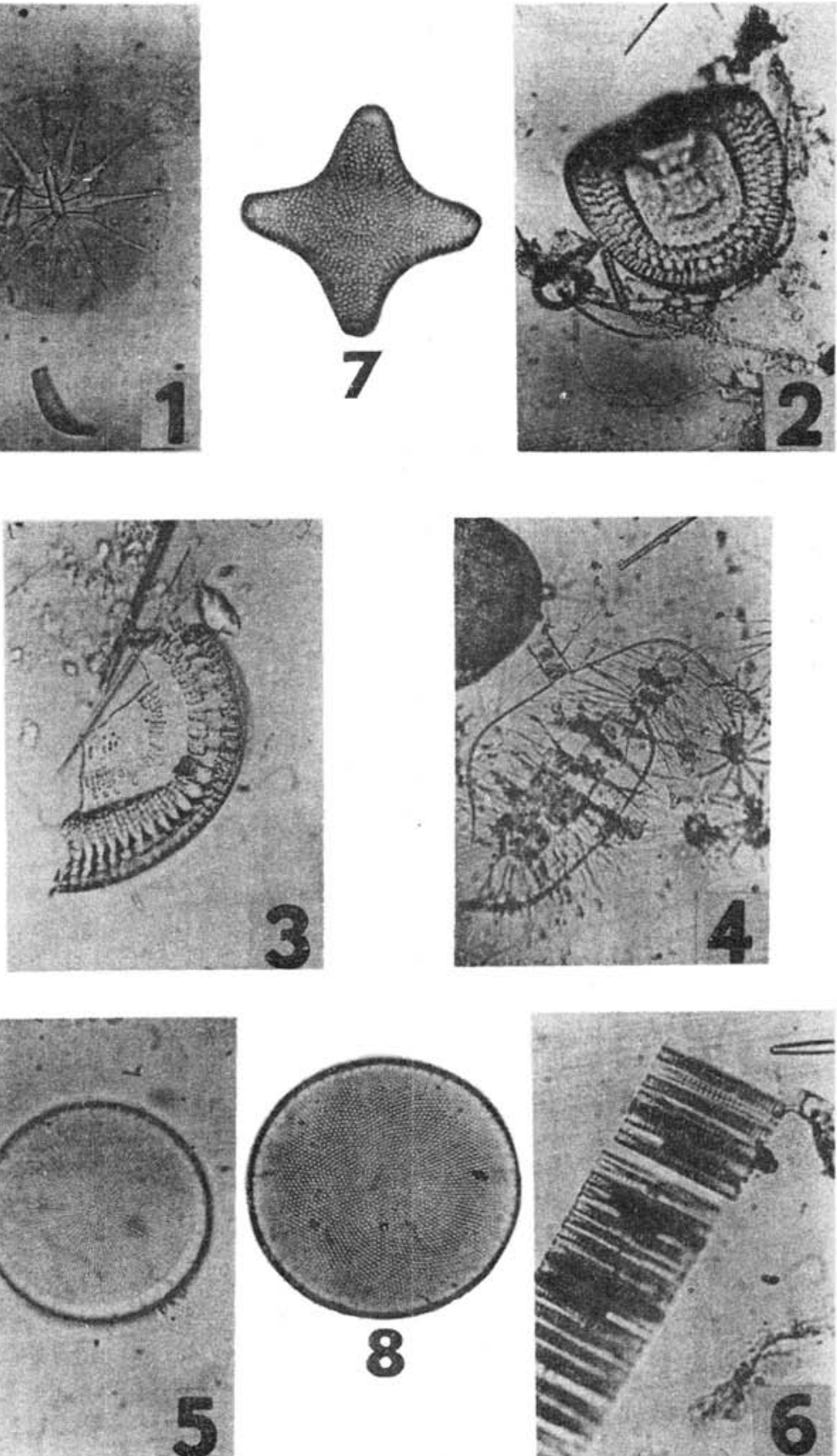

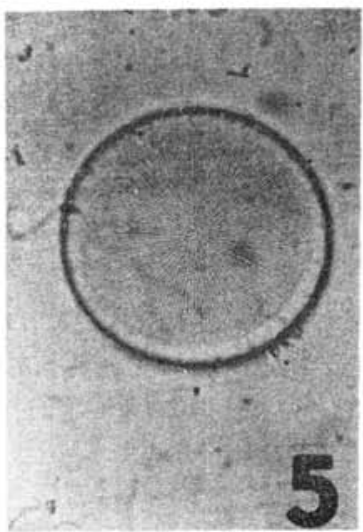


lámina II

Fig. 1 - A-Cymatosira Adaroi Azpeitia; B - Sceletonema costatum (Greville) Cleve.

Fig. 2 - Cymatosira Adaroi Azpeitia

Fig. 3 - Eupodiscus antiquus Cox

Fig. 4 - Hemidiscus ovalis Lohmann

Fig. 5 - Surirella Febigerii Lewis

Fig. 6 - Navicula Lyra Ehrenberg

Fig. 7 - Triceratiln pentacrinus (Ehr.) Wallich

Fig. 8 - Triceratium favus var. quadrata Ehrenberg

Fig. 9 - Triceratium formosum Brightwell 
MULLLER-MELCHERS, F.C. - Las Diatomeas del plancton...
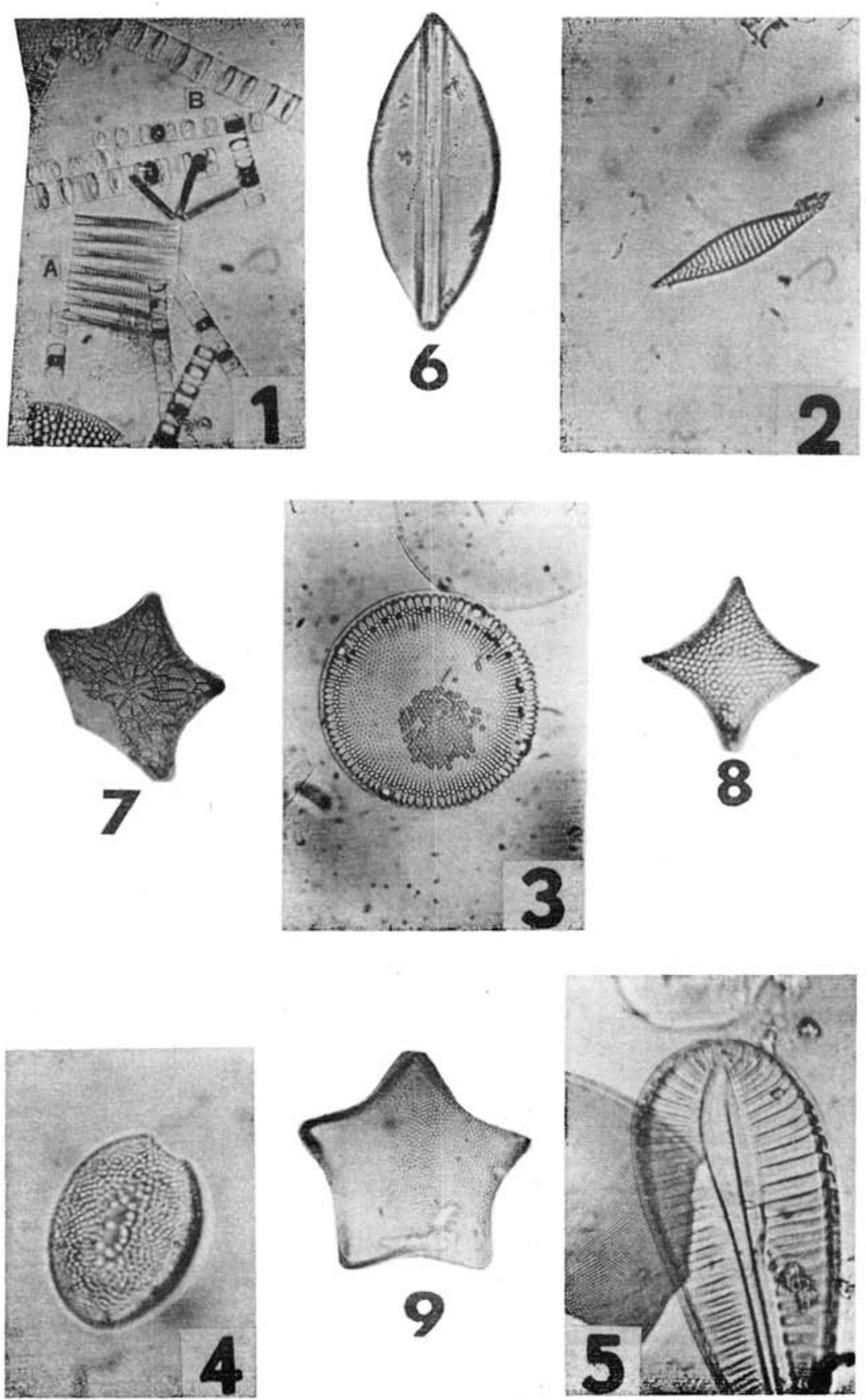